\title{
Influencia del genotipo y la temperatura sobre la carpelodia en papaya ${ }^{1}$
}

\section{Influence of genotype and temperature on carpellody of papaya}

\author{
Antonio Bogantes-Arias ${ }^{2}$,Eric Mora-Newcomer ${ }^{3}$
}

\begin{abstract}
Resumen
La carpelodia es un fenómeno que afecta las flores hermafroditas de la papaya y consiste en la transformación de los estambres en carpelos adicionales, que resulta en una malformación de los ovarios y consecuentemente de las frutas. El objetivo de este trabajo fue cuantificar la incidencia de la carpelodia en las flores de papaya, y el efecto de la temperatura sobre dicho fenómeno. El experimento se ejecutó entre octubre del 2006 y enero del 2007 en la Estación Experimental Los Diamantes, localizada en el cantón de Pococí, provincia de Limón, Costa Rica. Los tratamientos evaluados fueron cuatro líneas y cuatro híbridos en un diseño de bloques completos al azar con cuatro repeticiones. Las variables analizadas fueron el número de carpelos en las flores hermafroditas y las temperaturas diarias ocurridas durante los 60 días antes de la antesis de cada flor evaluada. El número de carpelos de las líneas y de los híbridos fue diferente durante cada semana ( $\mathrm{p} \leq 0,03)$, así como también en el promedio $(\mathrm{p}<0,0001)$ de las doce semanas del estudio. El cruce de una línea carpelódica con líneas de floración normal o ligeramente estériles tendió a producir híbridos con carpelodia, lo cual sugiere que es dominante. El estudio de correlación entre el número de carpelos y la temperatura indicó que la amplitud térmica durante el día fue un factor determinante en la inducción de la carpelodia.
\end{abstract}

Palabras clave: flor hermafrodita, carpelos, esterilidad femenina, amplitud térmica.

\begin{abstract}
Carpellody is a phenomenon that affects hermaphrodite flowers of papaya, and consists in the transformation of the stamens into additional carpels, resulting in ovary malformation that consequently affects fruit shape and diminishes its market value. The objective of this study was to quantify the incidence of flower carpellody in papaya, and the effect of temperature on this phenomenon. An experiment was carried out between October of 2006 and January of 2007 at "Los Diamantes" Agricultural Experiment Station, located in the province of Limón. Four breeding lines and four experimental hybrids were arranged in a randomized complete block design with four replications. The analyzed variables were the carpel number for ovaries of hermaphrodite flowers and the daily temperature during a 60-day period prior to floral anthesis of each evaluated flower. The carpel number of lines and hybrids differed significantly each week $(p \leq 0,03)$. Line and hybrid averages also differed significantly during the twelve-week period $(\mathrm{p}<0,0001)$. The cross between a strong carpellodic line and a stable or a weak female-sterile line resulted in a hybrid that exhibited carpellody, which suggests that carpellody is dominant. The correlation between carpel number and
\end{abstract}

\footnotetext{
Recibido: 5 de febrero, 2017. Aceptado: 14 de marzo, 2017. Este trabajo se realizó como parte del proyecto de Mejoramiento de Variedades Vegetales. Convenio entre la Universidad de Costa Rica (UCR) e Instituto Nacional de Innovación y Transferencia en Tecnología Agropecuaria (INTA), Costa Rica.

2 Instituto Nacional de Innovación y Transferencia en Tecnología Agropecuaria, San José, Costa Rica. abogantes@inta.go.cr

3 Universidad de Costa Rica, Estación Experimental Agrícola Fabio Baudrit Moreno, Alajuela, Costa Rica. eric.mora@ucr.ac.cr
} 
temperature suggests that the amplitude of temperature fluctuations during the day was a determinant factor in the induction of carpellody.

Keywords: hermaphrodite-flower, carpels, female-sterility, temperature amplitude.

\section{Introducción}

La papaya puede presentar tres tipos de plantas de acuerdo al sexo de sus flores. Las plantas hermafroditas presentan flores con ovarios de forma elongada con cinco carpelos, cinco rayos estigmáticos y diez estambres situados en dos series de cinco; sus frutas tienden a ser alargadas o en forma de pera, dependiendo de la variedad. Las plantas femeninas presentan flores con ovario elipsoidal liso, estigma grande y sin estambres; sus frutas normalmente son de forma redonda con una cavidad interna mayor que las hermafroditas. Por ese motivo, las frutas de las plantas hermafroditas normalmente tienen una mayor aceptación comercial (Nakasone y Lamoureux, 1982; Arkle y Nakasone, 1984; Chan et al., 2003; Gil y Miranda, 2005). En condición silvestre, la papaya es dioica y las plantas masculinas se caracterizan por presentar inflorescencias con cientos de flores con estambres y sin ovario (Arkle y Nakasone, 1984; Chan et al., 2003; Gil y Miranda, 2005).

La identidad sexual de la papaya está determinada por una serie de genes ligados, que se heredan como un solo locus. Estos genes codifican para los caracteres de estambres, ovarios y longitud de los pedúnculos de las inflorescencias. Su modelo plantea que existen tres alelos: el hermafrodita dominante (M2), el masculino dominante (M1) y el femenino recesivo (m), los cuales confieren los genotipos M2m, M1 m y mm, respectivamente. Los tres genotipos homocigotas dominantes son combinaciones letales (M1M1, M1M2 y M2M2) que nunca llegan a desarrollarse (Storey, 1953). Por lo anterior, las plantas hermafroditas y masculinas son heterocigotas obligados. Más recientemente, Liu et al. (2004) determinaron que en realidad la papaya presenta cromosomas sexuales (X y Y), lo cual explica las segregaciones descritas por Storey (1953).

Los casos registrados de anormalidades florales no son escasos y algunas de las más frecuentes son: filodia, sepalodia, petalodia, estaminodia y carpelodia (Meyer, 1966; Cruz y Alcántara, 2000). En el caso de papaya, la información existente sobre la biología reproductiva, ontogenia y estructura floral está centrada en condiciones de Hawai, India y Sudáfrica (Arkle y Nakasone, 1984; Ronse y Smets, 1999).

En las plantas hermafroditas de papaya existen dos problemas relacionados con la floración. El primero consiste en una disminución progresiva en el número de carpelos, fenómeno que se conoce como "esterilidad femenina" (Nakasone y Lamoureux, 1982). La disminución parcial a cuatro, tres o dos carpelos resulta en una fruta alargada y deformada, la cual pierde valor comercial (Silva et al., 2007a; Bogantes et al., 2011). Esta pérdida de carpelos va acompañada de la consecuente pérdida de estigmas y haces vasculares de los ovarios. En el caso más extremo, la pérdida de los cinco carpelos resulta en una flor con solo un vestigio de ovario (flor "vana"), similar a una flor masculina (Nakasone y Lamoureux, 1982).

El otro problema asociado a las flores hermafroditas consiste en la transformación de los estambres en carpelos adicionales, lo cual se conoce como carpelodia (Storey, 1953; Awada y Warren, 1957; Awada, 1958; Nakasone, 1967; Storey, 1967); esto resulta en una malformación de los ovarios y consecuentemente de las frutas. En casos extremos, cinco carpelos adicionales se fusionan completamente con los carpelos originales, y las flores que sufren este tipo de transformación se conocen como flores pentándricas (Nakasone, 1967). Las flores pentándricas originan frutos con surcos profundos en la pared del ovario y son similares a aquellos producidos por plantas femeninas, 
redondas, con una cavidad grande interna y surcos internos visibles, los cuales deprecian el valor comercial (Silva et al., 2007a; 2007b).

La carpelodia es un problema fisiológico, de expresión variable en el tiempo y es favorecido por las bajas temperaturas, déficit hídrico y deficiencias de nitrógeno (Awada y Warren, 1957; Nakasone, 1967). No obstante, se han observado diferencias importantes según la genética de los materiales (Cruz y Alcántara, 2000), al punto de que se ha convertido en una variable importante en la selección o adaptación de líneas e híbridos (Chan, 1984; Mora y Bogantes, 1999-2002; Rodríguez-Pastor, 2002; Silva et al., 2008; Mirafuentes-Hernández y SantamaríaBasulto, 2014). En Hawaii, Arkle y Nakasone (1984) determinaron que el ovario ya es susceptible a la inducción de la carpelodia dos semanas antes de que el primordio floral se vuelva visible al ojo humano. En esas condiciones subtropicales, el tiempo transcurrido entre el momento en que el primordio floral es visible al ojo humano y la antesis es de 53 a 63 días. Este periodo varía según localidades, probablemente como consecuencia de las diferentes temperaturas. En Bangladesh en flores femeninas dicho período es de 27-29 días (Azad y Rabbani, 2004). Sobre la papaya del cultivar "Solo" en Sudáfrica se menciona un lapso de 70 días (Sippel et al., 1989). En Venezuela, encontraron que ese tiempo fue de 30 días en el cultivar "Cartagena amarilla" y 33 días en el cultivar "Catagena roja” (Parés et al., 2002; 2004). En Tamaulipas, México, ese tiempo hasta antesis tardó entre 22 y 27 días (Vázquez et al., 2008).

Este estudio se llevó a cabo con el objetivo de cuantificar la incidencia de la carpelodia en las flores de papaya, y el efecto de la temperatura sobre dicho fenómeno.

\section{Materiales y métodos}

El experimento se llevó a cabo entre octubre del 2006 y enero del 2007 en la Estación Experimental Los Diamantes, localizada en el cantón de Pococí, provincia de Limón, Costa Rica. La altura del área de la investigación es de $175 \mathrm{msnm}$, y tiene una temperatura mínima promedio de $24{ }^{\circ} \mathrm{C}$ y una máxima promedio de $29{ }^{\circ} \mathrm{C}$. La precipitación promedio anual es de $4500 \mathrm{~mm}$.

Los tratamientos fueron cuatro líneas y cuatro híbridos producidos en conjunto entre la Universidad de Costa Rica (UCR) y el Instituto Nacional de Innovación y Transferencia de Tecnología Agropecuaria (INTA), y se describen en el Cuadro 1.

Cuadro 1. Líneas e híbridos de papaya utilizados como tratamientos para evaluar la manifestación de la carpelodia en la papaya (Carica papaya) del trópico húmedo. Guápiles, Limón, Costa Rica. 2006.

Table 1. Papaya lines and hybrids used as treatments in order to evaluated the manifestation of carpellody in papaya (Carica papaya) of the humid tropic. Guápiles, Limón, Costa Rica. 2006.

\begin{tabular}{cccc}
\hline Tratamiento & Línea & Tratamiento & Híbrido \\
\hline 1 & MC3-6 & 5 & MC3-6*HM3-7 \\
2 & MC3-8 & 6 & MC3-8*HM3-7 \\
3 & HM3-7 & 7 & MC3-6*HM3-4 \\
4 & HM3-4 & 8 & MC3-8*HM3-4 \\
\hline
\end{tabular}

Las cuatro líneas usadas (tratamientos 1-4) fueron seleccionadas por la estabilidad mostrada en sus características florales durante un proceso de autofecundación y selección de tres años.

Las líneas MC3-6 y MC3-8 son derivadas de una planta común. La línea MC3-8 se caracteriza por presentar una alta estabilidad de sus flores hermafroditas, debido a que, por lo general, presenta cinco carpelos, 
independientemente de las condiciones climáticas. Su línea hermana (MC3-6), tiene en cambio, una fuerte tendencia a producir flores carpelódicas. La línea HM3-7 se caracteriza por presentar una alta estabilidad floral, mientras que la línea HM3-4 produce normalmente flores con un ligero grado de esterilidad femenina, es decir, un poco menos de los cinco carpelos normales.

Los híbridos (tratamientos 5-8) se planearon con base en las características ya conocidas en la flor de las líneas progenitoras. Los cruzamientos se hicieron en el campo de manera controlada para garantizar la pureza de los híbridos, siguiendo procedimientos básicos para evitar la contaminación genética.

El diseño experimental consistió en cuatro bloques completos al azar con ocho tratamientos en cada bloque. Los tratamientos estuvieron constituidos por parcelas de cinco plantas sembradas a 2,5 m entre sí y 2,5 m entre surco. Las plantas fueron sexadas en forma visual a las ocho semanas de edad y se evaluaron siempre las dos plantas hermafroditas del centro de cada repetición.

\section{Variables evaluadas}

Número de carpelos de las flores hermafroditas: se contó el número de carpelos en el ovario de la flor abierta más joven, siempre en la misma planta. Para distinguir y contar los carpelos se consideró el número de ramificaciones del estigma, los cuales son proporcionales (Figura 1a). Adicionalmente, se hizo un corte transversal en la zona más ancha del ovario en el tercio superior donde se observaron los carpelos o sus respectivos haces dorsiventrales (Figura 1b).

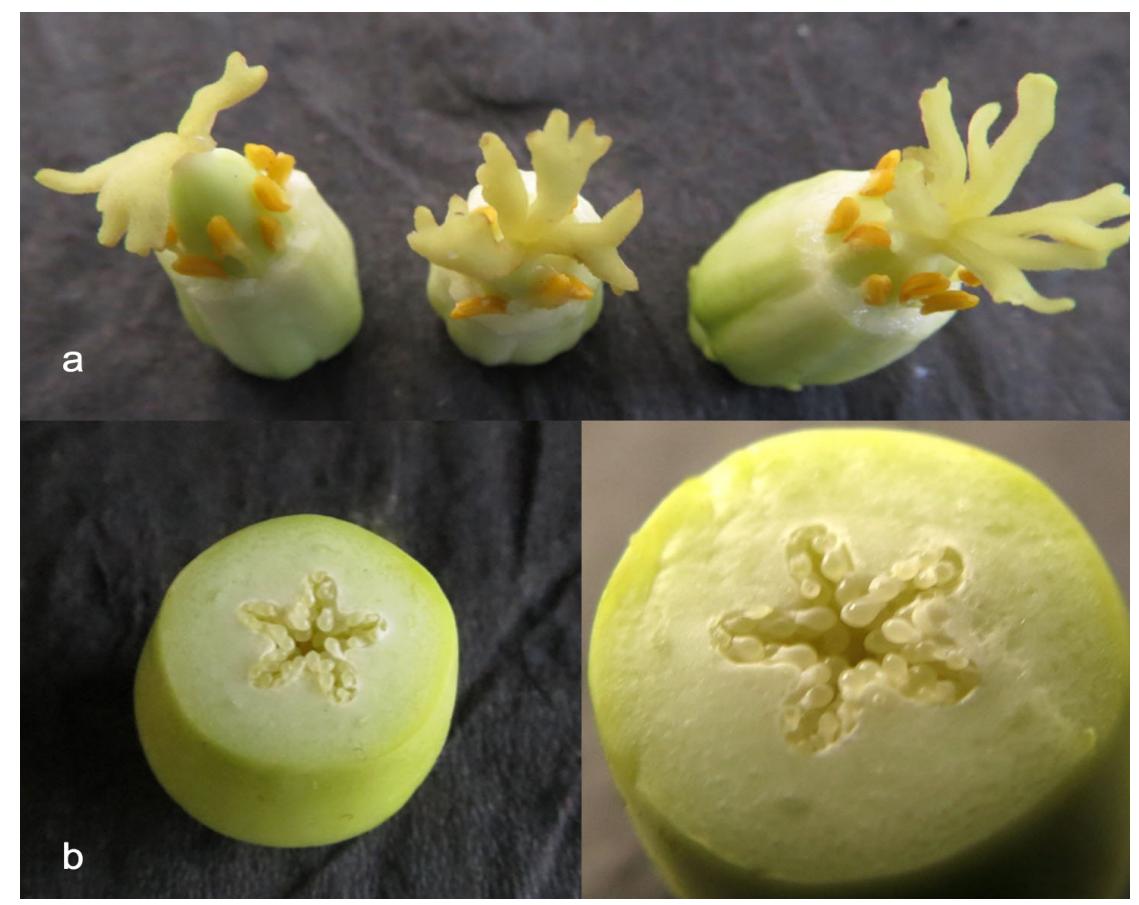

Figura 1. a) Ovarios de flor de papaya (Carica papaya) que presentan dos, cuatro y cinco ramificaciones del estigma. b) Ovarios que muestran cinco carpelos y sus respectivos haces dorsiventrales.

Figure 1. a) Ovaries of papaya (Carica papaya) flower showing two, four and five stigma branches. b) Ovaries containing five carpels and their marginal bundles. 
Por último, se consideraron los carpelos supernumerarios (por encima de cinco) adheridos al ovario (Figura 2b). Esta evaluación se inició cuando las plantas tenían tres meses y medio de trasplantadas al campo y se continuó durante doce semanas.

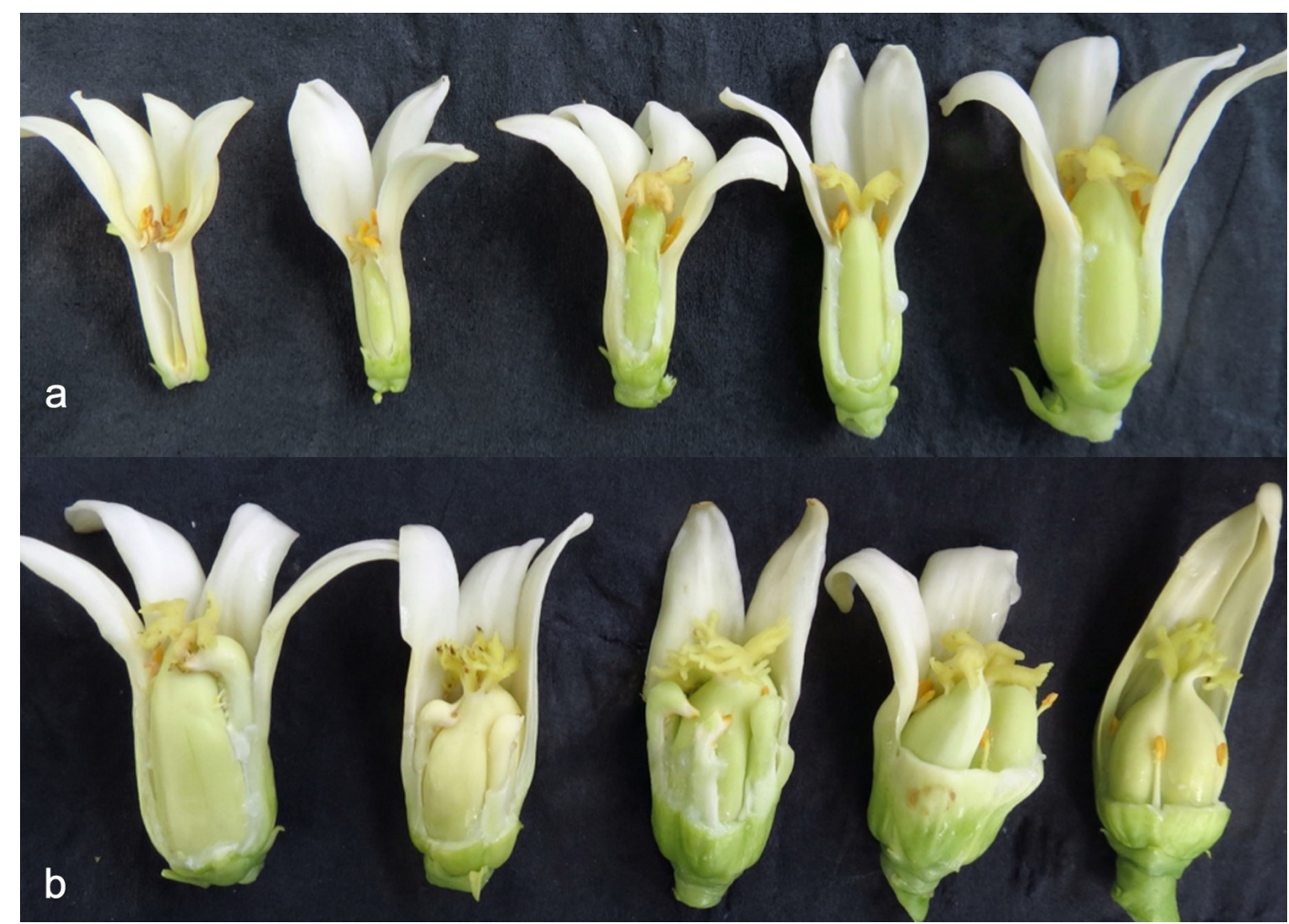

Figura 2. a) Secuencia de flores hermafroditas de papaya (Carica papaya) con diversos grados de esterilidad femenina, producto de la reducción en el número de carpelos desde 0 (extremo izquierdo) hasta 5 normales (extremo derecho). b) Secuencia de flores hermafroditas con carpelodia, desde un carpelo adicional (extremo izquierdo) hasta cinco carpelos adicionales o pentandria (extremo derecho). Guápiles, Costa Rica. 2006.

Figure 2. a) Papaya (Carica papaya) hermaphrodite flowers with varying degrees of female sterility as a result of the reduction in the number of carpels ranging from 0 (left) to 5 normal (right). b) Carpellody in hermaphrodite flowers, from an additional carpel (left) up to five additional carpels or pentandry (right). Guapiles, Costa Rica. 2006.

Temperatura: debido al reporte de que la tendencia a producir más de cinco carpelos es una respuesta a las bajas temperaturas (Nakasone, 1967), durante el período comprendido entre los 0 y 60 días antes de la antesis de cada flor evaluada, se recopilaron las temperaturas diarias ocurridas a intervalos de treinta minutos y registradas en la estación meteorológica de la Estación los Diamantes, ubicada a $10,21556^{\circ}$ latitud norte y $83,77288^{\circ}$ longitud oeste. Con esos datos se hicieron los siguientes tres grupos de interés para estudiar su posible relación con el número de carpelos en la flor.

a) Temperatura de la madrugada: agrupó todas las temperaturas ocurridas entre las doce media noche y las seis de la mañana. 
b) Amplitud térmica: este grupo consideró para cada día la diferencia entre la temperatura máxima y la mínima, la cual ha sido denominada como amplitud térmica (Peres-Martelleto et al., 2011).

c) Temperaturas menores a la media mínima: en este grupo se consideró todas las lecturas de temperatura diarias inferiores a los $21^{\circ} \mathrm{C}$ por día, que fue el promedio de la temperatura mínima durante el período del estudio.

Se realizó un análisis de varianza para la variable número de carpelos, complementado con su respectiva separación de medias. Además, se hicieron correlaciones ( $\mathrm{r}$ de Pearson) entre el número de carpelos de las flores hermafroditas con la temperatura de la madrugada, la amplitud térmica y las temperaturas menores a la media, correspondientes al " $x$ " día anterior a esa antesis, donde " $x$ " representa un día comprendido entre los 60 y 0 días anteriores a la antesis.

Todos los análisis fueron realizados utilizando el programa estadístico SAS System, versión 9 con el apoyo del respectivo manual para el análisis de experimentos agrícolas (López y López, 1995).

\section{Resultados}

\section{Carpelodia}

El número de carpelos de las líneas y de los híbridos fue diferente durante cada semana ( $\mathrm{p} \leq 0,03)$, y en el promedio $(\mathrm{p}<0,0001)$ de las doce semanas del estudio (Cuadro 2$)$.

Cuadro 2. Número de carpelos por ovario de cada semana y promedio de las doce semanas en la flor abierta más joven de distintas líneas e híbridos de papaya (Carica papaya L.). Guápiles, Limón. Costa Rica. 2007.

Table 2. Number of carpels per ovary in the youngest opened flower, and average for the twelve-week period for different papaya (Carica papaya) lines and hybrids. Guápiles, Limón. Costa Rica. 2007.

\begin{tabular}{ccccccccc}
\hline \multirow{2}{*}{ emana } & \multicolumn{9}{c}{ Líneas } & \multicolumn{3}{c}{ Híbridos } \\
\cline { 2 - 8 } & MC3-6 & MC3-8 & HM3-7 & HM3-4 & $\begin{array}{c}\text { MC3-6 } \\
\text { *HM3-7 }\end{array}$ & $\begin{array}{c}\text { MC3-8 } \\
\text { *HM3-7 }\end{array}$ & $\begin{array}{c}\text { MC3-6 } \\
\text { *HM3-4 }\end{array}$ & $\begin{array}{c}\text { MC3-8 } \\
\text { *HM3-4 }\end{array}$ \\
\hline 1 & $7,62 \mathrm{a}$ & $4,38 \mathrm{~b}$ & $5,00 \mathrm{~b}$ & $3,12 \mathrm{~b}$ & $8,12 \mathrm{a}$ & $4,75 \mathrm{~b}$ & $5,00 \mathrm{~b}$ & $4,87 \mathrm{~b}$ \\
2 & $9,00 \mathrm{~b}$ & $4,87 \mathrm{c}$ & $5,00 \mathrm{c}$ & $3,88 \mathrm{c}$ & $9,87 \mathrm{a}$ & $5,00 \mathrm{c}$ & $7,88 \mathrm{~b}$ & $4,88 \mathrm{c}$ \\
3 & $8,50 \mathrm{a}$ & $4,12 \mathrm{bc}$ & $5,00 \mathrm{bc}$ & $3,75 \mathrm{c}$ & $6,00 \mathrm{~b}$ & $4,88 \mathrm{bc}$ & $5,38 \mathrm{bc}$ & $4,25 \mathrm{bc}$ \\
4 & $7,62 \mathrm{a}$ & $3,87 \mathrm{c}$ & $5,00 \mathrm{bc}$ & $4,25 \mathrm{c}$ & $7,25 \mathrm{ab}$ & $4,75 \mathrm{c}$ & $6,12 \mathrm{abc}$ & $5,00 \mathrm{bc}$ \\
5 & $9,88 \mathrm{a}$ & $5,00 \mathrm{~b}$ & $5,00 \mathrm{~b}$ & $4,75 \mathrm{~b}$ & $10,00 \mathrm{a}$ & $5,00 \mathrm{~b}$ & $9,37 \mathrm{a}$ & $5,00 \mathrm{~b}$ \\
6 & $9,88 \mathrm{a}$ & $4,50 \mathrm{~b}$ & $4,88 \mathrm{~b}$ & $4,00 \mathrm{~b}$ & $8,75 \mathrm{a}$ & $4,62 \mathrm{~b}$ & $8,00 \mathrm{a}$ & $4,62 \mathrm{~b}$ \\
7 & $8,88 \mathrm{a}$ & $4,88 \mathrm{~b}$ & $4,88 \mathrm{~b}$ & $4,25 \mathrm{~b}$ & $9,00 \mathrm{a}$ & $4,75 \mathrm{~b}$ & $8,00 \mathrm{a}$ & $5,00 \mathrm{~b}$ \\
8 & $8,50 \mathrm{a}$ & $4,37 \mathrm{bc}$ & $5,00 \mathrm{bc}$ & $4,25 \mathrm{c}$ & $6,5 \mathrm{ab}$ & $4,50 \mathrm{bc}$ & $6,12 \mathrm{bc}$ & $4,62 \mathrm{bc}$ \\
9 & $9,38 \mathrm{a}$ & $4,38 \mathrm{~d}$ & $5,00 \mathrm{~cd}$ & $5,00 \mathrm{~cd}$ & $7,25 \mathrm{~b}$ & $4,88 \mathrm{~cd}$ & $6,38 \mathrm{bc}$ & $5,00 \mathrm{~cd}$ \\
10 & $9,62 \mathrm{a}$ & $4,12 \mathrm{c}$ & $5,00 \mathrm{c}$ & $4,87 \mathrm{c}$ & $9,62 \mathrm{a}$ & $4,88 \mathrm{c}$ & $7,88 \mathrm{~b}$ & $5,00 \mathrm{c}$ \\
11 & $8,25 \mathrm{a}$ & $3,75 \mathrm{c}$ & $4,88 \mathrm{bc}$ & $4,75 \mathrm{bc}$ & $6,50 \mathrm{ab}$ & $3,75 \mathrm{c}$ & $5,12 \mathrm{bc}$ & $4,88 \mathrm{bc}$ \\
12 & $7,12 \mathrm{a}$ & $3,75 \mathrm{bc}$ & $4,88 \mathrm{bc}$ & $3,62 \mathrm{c}$ & $5,88 \mathrm{ab}$ & $3,12 \mathrm{c}$ & $5,25 \mathrm{abc}$ & $4,88 \mathrm{bc}$ \\
Promedio & $8,69 \mathrm{a}$ & $4,33 \mathrm{c}$ & $4,96 \mathrm{c}$ & $4,21 \mathrm{c}$ & 7,90 & $4,57 \mathrm{c}$ & $6,71 \mathrm{~b}$ & $4,83 \mathrm{c}$ \\
\hline
\end{tabular}

${ }^{1}$ Tratamientos con diferente letra en una misma fila presentan diferencias significativas según prueba de Tukey al 5\%/ treatments with different letter in the same line present significant differences according Tukey test at 5\%.

La línea HM3-7 mostró un promedio de 4,96 carpelos por ovario, mantuvo flores de cinco carpelos durante casi todas las doce semanas de la evaluación y fue diferente a la MC3-6 (Cuadro 2) (Figura 3a). 


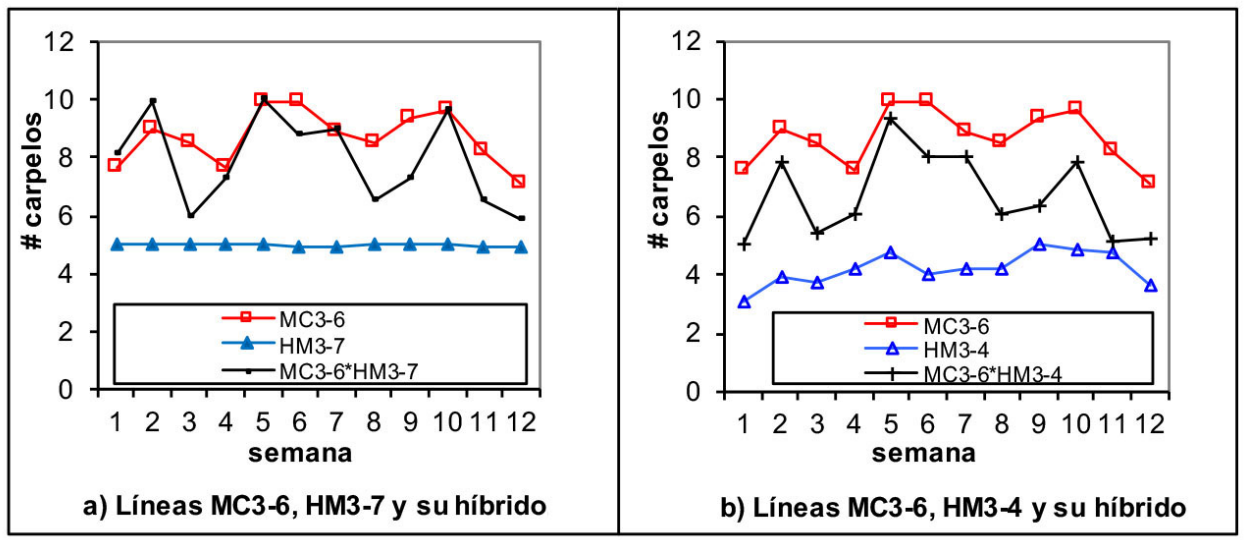

Figura 3. Número de carpelos en las líneas MC3-6, HM3-7, HM3-4 (a, b) de papaya (Carica papaya) y sus respectivos híbridos (a, b). Guápiles, Costa Rica. 2007.

Figure 3. Number of carpels in papaya's MC3-6, HM3-7, HM3-4 (Carica papaya) lines (a, b) and their respective hybrids (a, b). Guapiles, Costa Rica. 2007.

La MC3-6 fue la línea con el mayor promedio $(8,69)$ de carpelos por ovario durante las doce semanas (Cuadro 2). Los ovarios de sus flores tuvieron un número de carpelos por encima de seis, según el recuento hecho por semana (Cuadro 2, Figuras 3a y b). El aumento en ese número de carpelos fue parcial en las primeras semanas (Figuras 3 y 2b), pero durante las semanas cinco, seis, nueve y diez, las flores de esta línea alcanzaron un valor de diez o cercano a los diez carpelos (Figuras 3 a, b y $2 b$ ).

La línea HM3-4 tuvo un promedio de 4,21 carpelos por ovario en las doce semanas (Cuadro 2) con una tendencia general a producir flores con menos de cinco carpelos en su ovario (Cuadro 2, Figuras 2a y 4d). La línea MC3-8 presentó un promedio de 4,33 carpelos por ovario, lo cual significa un 100\% menos de carpelos que su línea hermana MC3-6 (Cuadro 2). La tendencia de esta línea fue similar a la HM3-4 en producción de flores con menos de cinco carpelos durante todo el tiempo del estudio (Cuadro 2, Figura 4c y d).

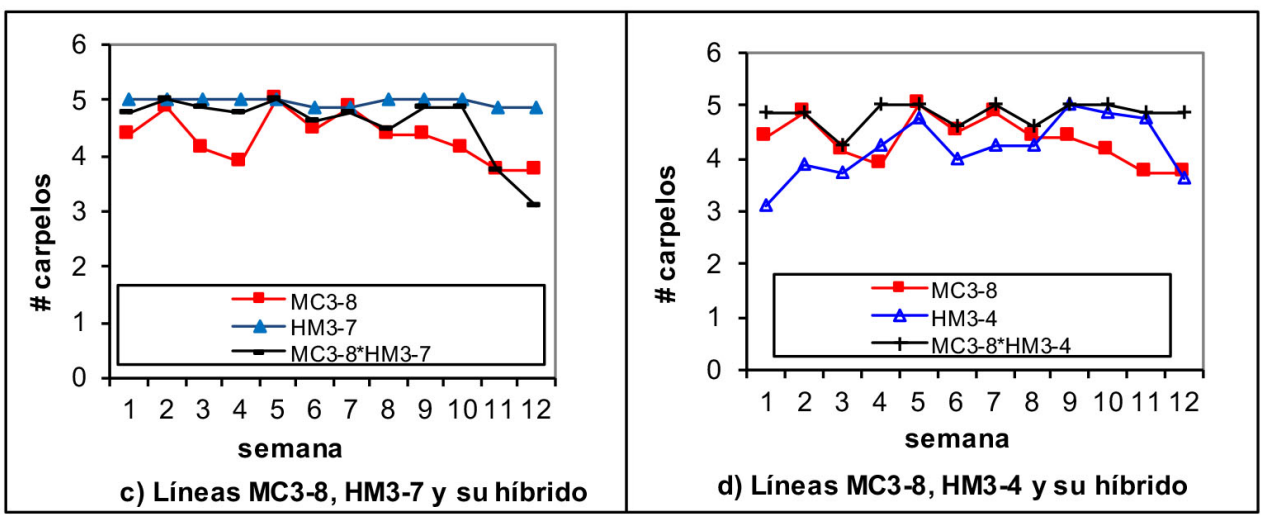

Figura 4. Número de carpelos en las líneas MC3-8, HM3-7 y HM3-4 (c, d) de papaya (Carica papaya) y sus respectivos híbridos (c, d). Guápiles, Limón, Costa Rica. 2007.

Figure 4. Number of carpels in papaya's MC3-8, HM3-7, HM3-4 (Carica papaya) lines (c, d) and their respective hybrids (c, d). Guápiles, Limón, Costa Rica. 2007. 
El número promedio de carpelos del híbrido originado por el cruce entre la línea progenitora altamente carpelódica (MC3-6) con la línea estable (HM3-7) fue el más alto de todos $(7,90)$, con un promedio similar a la primera $(8,69)$ (Cuadro 2). Además, durante las semanas dos, cinco, seis, siete y diez fue cuando se expresó la mayor carpelodia de este híbrido (Figura 3a). Además, el número promedio de carpelos en la flor del híbrido originado por la línea más carpelódica (MC3-6) y la línea estéril (HM3-4), fue diferente a cada uno de sus padres, pero muy similar al promedio de esos progenitores $(6,45)$; un poco más cercano al progenitor carpelódico pero sin ser significativamente igual (Cuadro 2). También, ese comportamiento fue similar en las evaluaciones semanales (Figura 3 b).

El híbrido entre la línea estable (HM3-7) con la línea estéril (MC3-8), mostró una tendencia a presentar un número de carpelos por debajo de cinco, con un promedio muy similar al de los padres $(4,64)$, sin diferir significativamente de ninguno de ellos durante las doce semanas (Cuadro 2, Figura 4c).

El híbrido entre las líneas estériles MC3-8 x HM3-4, también mostró un promedio en el número de carpelos por debajo de cinco, con una tendencia a la esterilidad similar a sus padres (Cuadro 2, Figura 4d).

\section{Efecto de la temperatura}

Los datos de temperatura durante el período del estudio, indicaron una máxima absoluta de $32,0{ }^{\circ} \mathrm{C}$ con un promedio de $29,0{ }^{\circ} \mathrm{C}$, y una mínima absoluta de $17,5^{\circ} \mathrm{C}$ con un promedio de $21,0{ }^{\circ} \mathrm{C}$.

En este estudio, las correlaciones significativas para carpelodia antes de la apertura floral variaron según el grupo de temperatura utilizada. La correlación entre la amplitud térmica con el número de carpelos en flor fue la que obtuvo el mayor número (23) de correlaciones significativas. El valor $\mathrm{r}$ de la correlación entre la amplitud térmica y el número de carpelos varió de 0,53 a 0,81 con una relación positiva (Cuadro 3).

La temperatura de la madrugada correlacionó significativamente en siete ocasiones con carpelodia, y temperaturas menores a la media correlacionó significativamente en ocho ocasiones. El valor $\mathrm{r}$ de la correlación entre temperatura de la madrugada con el número de carpelos varió de 0,54 a 0,74 con una relación negativa, y el de las temperaturas menores a la media varió de 0,57 a 0,75 (Cuadro 4).

\section{Discusión}

\section{Carpelodia}

En las líneas, sus flores se comportaron de acuerdo con sus características observadas en fases previas con respecto al número de carpelos. La alta estabilidad floral de la línea HM3-7 significa la presencia siempre de una típica flor elongada, la más común en las flores andromonoicas, con cinco carpelos y diez estambres en dos series de cinco, situados al final del tubo de la corola (Nakasone y Lamoureux, 1982; Gil y Miranda, 2005). Su ovario alargado es el que le da la forma final a la fruta comercial de la papaya.

Contrario a la estabilidad de la línea HM3-7, el comportamiento de la línea MC3-6 con un alto promedio de carpelos en el tiempo, es típico de flores con mucha carpelodia; en ese sentido, la carpelodia también puede ser parcial (Storey, 1967) (Figura 2b), con un resultado final de fruta deformada y sin valor comercial (Silva et al., 2007a). Además, los picos de alta carpelodia mostrados por esta línea durante las semanas cinco, seis, nueve y diez convierten sus flores hermafroditas en flores pentándricas (Figura 2b) con frutos del mismo nombre que se caen y tampoco tienen valor comercial (Nakasone, 1967; Silva et al., 2007a). En islas Canarias, Rodríguez-Pastor (2002) coincide con el reporte de cantidades de hasta 60 frutos por planta con carpelodia en el cultivar "Baixinho de Santa 
Cuadro 3. Líneas e híbridos de papaya (Carica papaya), con correlaciones entre amplitud térmica con el número de carpelos en la flor abierta más joven durante sesenta días preantesis. Guápiles, Limón, Costa Rica. 2007.

Table 3. Lines and hybrids of papaya (Carica papaya), with correlations between temperature amplitude and number of carpels in the youngest opened flower, with 60 days of preanthesis. Guápiles, Limón, Costa Rica. 2007.

\begin{tabular}{|c|c|c|c|c|c|}
\hline \multirow[t]{2}{*}{ Daa } & \multicolumn{2}{|c|}{ Líneas } & \multicolumn{3}{|c|}{ Híbridos } \\
\hline & MC3-6 & MC3-8 & MC3-6*HM3-7 & MC3-8*HM3-7 & MC3-6*HM3-4 \\
\hline 5 & & & & $0,61^{*}$ & \\
\hline 25 & & $0,54 *$ & & & \\
\hline 29 & & & $0,53^{*}$ & $0,66^{*}$ & \\
\hline 34 & & & & $0,70 * *$ & \\
\hline 38 & & & & $0,63^{*}$ & \\
\hline 39 & & & & $0,53^{*}$ & \\
\hline 41 & & & & $0,78 * *$ & \\
\hline 42 & & & & $0,63^{*}$ & \\
\hline 43 & & & & $0,58^{*}$ & \\
\hline 45 & $0,53^{*}$ & & & $0,68 * *$ & \\
\hline 46 & $0,57^{*}$ & & & & \\
\hline 47 & & & & $0,68 * *$ & \\
\hline 48 & $0,68 * *$ & $0,59 *$ & $0,56^{*}$ & $0,79 * *$ & $0,59^{*}$ \\
\hline 49 & & & & $0,81 * *$ & \\
\hline 50 & & & & $0,57 * *$ & \\
\hline 58 & $0,59 *$ & & & $0,63^{*}$ & \\
\hline 60 & & & & & \\
\hline
\end{tabular}

* $\mathrm{P} \leq 0,05 * * \mathrm{P} \leq 0,01$ Daa: días antes de la antesis. El área sombreada resalta las correlaciones en los días preantesis dentro o muy cerca del período crítico de diferenciación floral establecido (43-25 días). ( $\mathrm{n}=12$, promedio de 96 observaciones) / $* \mathrm{P} \leq 0,05 * * \mathrm{P} \leq 0,01$, Daa: days before antesis. The shaded area highlights the correlations in preantesis days within or very close to the critical period of established floral differentiation (43-25 days). ( $n=12$, average of 96 observations).

Amalia" en el primer ciclo productivo. No obstante, Chan (1984) y Rodríguez-Pastor (2002), encontraron que se puede dar una disminución de la carpelodia con el incremento en la edad de las plantas.

Contrario a la carpelodia, en las flores de las líneas MC3-8 y HM3-4 lo que se obtuvo fue la pérdida de carpelos, característica que se relaciona más con la esterilidad femenina (Nakasone y Lamoureux, 1982) (Figura 2a). Este fenómeno, cuando está relacionado con la pérdida de dos o más carpelos en el ovario (Figura 2a), se considera severo y en la mayoría de los casos sus frutas no tienen valor comercial (Bogantes et al., 2011).

Esas tendencias opuestas y tan marcadas en la producción de carpelos de varias de las líneas parentales evaluadas, condujeron también el comportamiento de sus híbridos. En ese sentido, fue evidente que el número promedio de carpelos del híbrido originado por el cruce entre la línea progenitora altamente carpelódica (MC3-6) con la línea estable (HM3-7) tuvo un comportamiento similar al primero, lo cual sugiere una dominancia parcial hacia la tendencia carpelódica. Este efecto fue más notorio en las semanas dos, cinco, seis, nueve y diez, cuando se expresó la mayor carpelodia (Cuadro 2, Figura 3a). En Brasil, Silva et al. (2008) reportaron una fuerte expresión de la carpelodia en plantas de los genotipos 52BC1S1 y BC2, insinuaron que es por herencia de uno de sus padres, reportado como Cariflora, pero dicha comparación no fue realizada; por otra parte, Chan (1984) estimó una heredabilidad (h2) de este fenómeno en un $82 \%$. 
Cuadro 4. Líneas e híbridos de papaya (Carica papaya), con correlaciones entre temperatura de la madrugada (negrita) y temperaturas menores a la media con el número de carpelos en la flor abierta más joven durante sesenta días preantesis. Guápiles, Limón, Costa Rica. 2007.

Table 4. Papaya (Carica papaya) line and hybrids, with correlations between temperature at dawn (bold) and below-average temperature, with carpel numbers of the youngest opened flower, with 60 days of preanthesis. Guápiles, Limón, Costa Rica. 2007.

\begin{tabular}{|c|c|c|c|c|c|c|}
\hline \multirow{2}{*}{ Daa } & \multicolumn{3}{|c|}{ Líneas } & \multicolumn{3}{|c|}{ Híbridos } \\
\hline & MC3-6 & MC3-8 & HM3-4 & MC3-6*HM3-7 & MC3-6*HM3-4 & MC3-8*HM3-4 \\
\hline 2 & & & $\begin{array}{c}\mathbf{- 0 , 7 2} * * \\
0,75 * *\end{array}$ & & & \\
\hline 7 & & & $\begin{array}{c}\mathbf{- 0 , 5 4} * \\
0,58^{*}\end{array}$ & & & \\
\hline 13 & & & $0,59 *$ & & & \\
\hline 16 & $\begin{array}{l}-0,56^{*} \\
0,66^{*}\end{array}$ & & & $0,61 *$ & & \\
\hline 17 & & $0,63^{*}$ & & & & \\
\hline 25 & & & & & & \\
\hline 26 & & & $-0,74 * *$ & & & \\
\hline 36 & & & & $-0,60 *$ & $-0,55^{*}$ & \\
\hline 40 & & & & $0,57 *$ & & \\
\hline 41 & & & & & & $-0,65^{*}$ \\
\hline 48 & & & $0,58 *$ & & & \\
\hline 50 & & & & & & \\
\hline 60 & & & & & & \\
\hline
\end{tabular}

* $\mathrm{P} \leq 0,05, * * \mathrm{P} \leq 0,01$. Daa: días antes de la antesis. El área sombreada resalta las correlaciones en los días preantesis dentro o muy cerca del período crítico de diferenciación floral establecido (43-25 días). ( $\mathrm{n}=12$, promedio de 96 observaciones) / $\mathrm{P} \leq 0,05$, ** $\mathrm{P} \leq 0,01$. Daa: days before antesis. The shaded area highlights the correlations in preantesis days within, or very close to the critical period of established floral differentiation (43-25 days). ( $n=12$, average of 96 observations).

Cuando el híbrido fue originado por la línea carpelódica (MC3-6) pero con la línea estéril (HM3-4), el comportamiento en carpelodia fue más parecido al promedio de sus progenitores, lo cual marca una tendencia más intermedia en el número de carpelos producidos en la flor (Cuadro 2, Figura 3b).

En Brasil, Corrêa et al. (2008) obtuvieron dos híbridos con un mayor número de anormalidades en la flor por carpelodia, a partir del cruce entre materiales del grupo Solo Formosa, así como un híbrido (SS783 x JS12) que no presentó anormalidades florales, pero no analizaron la relación carpelódica con sus parentales. En el sureste de México, destacan al híbrido MSXJ por la baja carpelodia de sus flores comparadas con las de la papaya comercial Maradol (Mirafuentes-Hernández y Santamaría-Basulto, 2014).

\section{Efecto de la temperatura}

En este estudio, la amplitud térmica con una mayor cantidad de correlaciones, explicó entre 53 a $81 \%$ de la variación (r) en el número de carpelos (Cuadro 3). Otros factores variados y ya conocidos podrían influir en el resto de la diferenciación de los carpelos, a saber, el estado nutricional de la planta (especialmente nitrógeno), su estado hídrico, la cantidad de fruta presente (Awada y Warren, 1957; Chan, 1984), así como una interacción entre ellas y todas ellas con la temperatura (Cruz y Alcántara, 2000). 
Se relaciona la influencia de la temperatura, tanto alta como baja, con esterilidad y carpelodia respectivamente, pero se presume que la amplitud térmica (diferencia entre máxima y mínima) afectó la carpelodia solo en un 22\% de las flores (Terra-De-Almeida et al., 2003). También Silva et al. (2007 a) en Brasil atribuyeron más deformación de fruta por carpelodia en invierno, debido a la baja temperatura y su relación con la amplitud térmica (temperatura máximas y mínimas del día), diferencia que fue mayor en invierno por $2,1^{\circ} \mathrm{C}$. Cancela-Ramos et al. (2011), también en Brasil, observaron más carpelodia en la primavera y en el invierno por una mayor influencia de temperaturas bajas y suaves en los materiales genéticos que ellos consideran como más sensibles a la expresión de este rasgo.

En el presente caso, también es probable que esta correlación no sea más alta en vista de la existencia de un "límite inferior" de temperatura a la cual se induzca la carpelodia, es decir, variaciones por encima de este límite no afectarían el número de carpelos y por lo tanto no serían detectados como fuentes de variación en la correlación. No obstante, siempre en Brasil y en condiciones de invernadero, Peres-Martelleto et al. (2011) estimaron una correlacion lineal $(\mathrm{r}=0,60)$ entre amplitud térmica mensual y frutos carpeloides de papaya. En su caso plantearon la hipótesis de que temperaturas más elevadas y un mayor vigor de la planta representan un peso significativo sobre la formación de frutos con carpelodia en flores hermafroditas normales.

El estudio más detallado sobre la dinámica y la estimación del efecto de la temperatura sobre la carpelodia en esos días preantesis fue el de Arkle y Nakasone (1984) hecho en Hawaii; ellos reportaron en esas condiciones que el primordio floral se vuelve visible al ojo humano entre 56 y 63 días antes de la antesis, mientras que el tiempo transcurrido entre diferenciación floral y antesis es de 63-70 días. Además, establecieron para ese clima que las temperaturas frías pueden inducir carpelodia en la flor desde los 70 hasta los 41 días antes de la antesis.

Previo a este estudio, en la misma zona y con materiales parientes a los usados en el presente experimento, se determinó que las flores en esas plantas de papaya eran visibles al ojo entre 34 y 40 días antes de la antesis, es decir, un $38 \%$ menos de tiempo que en Hawai (estudio tomado como referencia). Si se extrapola dicha diferencia es probable que el ciclo total desde diferenciación hasta antesis sea, en condiciones de Costa Rica, de 39 a 43 días. Entonces el período de tiempo en el cual la temperatura podría afectar la carpelodia en las condiciones de este estudio, se hipotetizó entre 43 y 25 días antes de la antesis o apertura floral. Dicha hipótesis se volvió relevante porque con los datos obtenidos se puede observar (Cuadro 3) que de las veintitres correlaciones entre amplitud térmica con los cambios en el número de carpelos, diecinueve se presentaron en el período comprendido entre 49 y 25 días preantesis, dentro o muy cercano a lo que se había estimado por aproximación (43-25 días) como período en el cual se podría considerar que cambios en la temperatura afectarían la carpelodia. Durante ese lapso (25 a 49 días preantesis) la amplitud térmica menor fue de $2,3{ }^{\circ} \mathrm{C}$ y la mayor de $12,3{ }^{\circ} \mathrm{C}$.

Solo cuatro correlaciones se ubicaron fuera del periodo establecido, una de las cuales, fue a cinco días antes de la antesis y podría ser producto del azar, debido a que con esa edad ya los carpelos estaban formados.

El día 48 antes de la antesis fue el de mayores correlaciones entre carpelodia y amplitud térmica. Se dio en dos líneas y tres híbridos (62\% de los tratamientos), y una de esas líneas en las que hubo correlación fue la MC3-6.

La diferencia entre la temperatura máxima y la mínima 48 días antes de que se abrieran las flores de la MC3-6, correspondientes a las evaluaciones de las semanas dos, cinco, seis y nueve, estuvo entre 10,2 y $11,2{ }^{\circ} \mathrm{C}$. En estas semanas, como ya se comentó, las flores de las plantas en esa línea tuvieron alrededor de los diez carpelos en flor (Cuadro 2, Figura 3a).

El factor térmico y la carpelodia fueron dos parámetros que sumaron cerca del $83 \%$ de las correlaciones significativas dentro o muy cerca del periodo crítico de diferenciación floral establecido (43-25 días), lo cual, no sucedió cuando se correlacionó la temperatura de la madrugada ni las temperaturas menores a la media.

Las correlaciones entre carpelodia con el factor térmico coinciden también con observaciones que los autores han hecho en plantaciones de Costa Rica (Parrita y Guanacaste), en donde la carpelodia observada coincide mucho con esos días de temperaturas por encima de los $30^{\circ} \mathrm{C}$, y por las noches bajan a menos de $20^{\circ} \mathrm{C}$ (amplitud térmica), y afecta tanto al híbrido Pococí como a la variedad criolla conocida como Lucia. 
En México, Mirafuentes-Hernández y Santamaría-Basulto (2014) destacaron que temperaturas máximas de 32 a $42{ }^{\circ} \mathrm{C}$ en el sureste del país, inciden en la producción de frutos pentándricos (máxima expresión de la carpelodia) y frutos con carpelodia parcial en plantas de la variedad Maradol.

\section{Conclusiones}

En términos generales y desde el punto de vista del mejoramiento genético, la utilidad práctica de estos resultados radica en la posibilidad de utilizar líneas parentales de buenas características agronómicas y comerciales, pero con problemas en su estabilidad floral.

Se puede concluir que el cruce de líneas con mucha diferencia en el número de carpelos producen híbridos con una tendencia a desarrollar un número de carpelos intermedios a ambos padres. Este resultado en particular, puede aportar un avance importante en cuanto a la disminución de la carpelodia, una línea con problemas por alta tendencia a la carpelodia podría producir un híbrido con buena estabilidad floral (pentámero) si se escoge a otro padre que presente una tendencia hacia la esterilidad (pocos carpelos). También se prevería que una línea carpelódica puede ser muy dominante en el número de carpelos de su híbrido cuando se cruza con una línea muy estable.

Con respecto al efecto de la temperatura se puede concluir que, cuando las diferencias diarias entre bajas y altas temperaturas son superiores a los $10{ }^{\circ} \mathrm{C}$, pueden causar una disminución en la producción que sería proporcional al número de flores afectadas con carpelodia. Además, los datos de este estudio se pueden utilizar para estimar el momento en que la amplitud térmica podría incidir negativamente en la producción. Esas pérdidas se podrían considerar entre 170 a 198 días después de ocurrido el evento. Lo anterior porque, en condiciones de Costa Rica, el período entre antesis y madurez de colecta varía de 145 a 155 días, y a ese dato se le suman los 25-43 días considerados en este estudio como período crítico de diferenciación floral en que la flor puede ser afectada.

\section{Literatura citada}

Arkle, T.D., and H.Y. Nakasone. 1984. Floral differentiation in the hermafroditic papaya. HortSci. 19:832-834.

Awada, M. 1958. Relationships of minimum temperature and growth rate with sex expression of papaya plants (Carica papaya L.). Tech. Bull. 38. Hawaii Univ. Agric. Exp. Sta., HI, USA.

Awada, M., and S.I. Warren. 1957. Effects of water and nitrogen application on composition, growth, sugars in fruits, yield, and sex expression of the papaya plants (Carica papaya L.). Tech. Bull. 33. Hawaii Univ. Agric. Exp. Sta., HI, USA.

Azad, M.A.K., and M.G. Rabbani. 2004. Studies on floral biology of different Carica species. Pakistán J. Biol. Sci. 7:301-304.

Bogantes, A., E. Mora, G. Umaña, y C. Loría. 2011. Guía para el cultivo de papaya en Costa Rica. MAG/UCR/INTA, San José, CRC.

Chan, Y.K. 1984. Studies on carpellody of stamens in papaya (Carica papaya). MARDI Res. Bull. Malaysia 12:157-162.

Chan, T.C., C.R. Yen, L.S. Chang, C.H. Hsiao, and S. Ko. 2003. All hermaphrodite progeny are derived by self-pollinating the sunrise papaya mutant. Plant Breed. 122:431-434.

Cancela-Ramos, H.C., M. Gonzaga-Pereira, F. Filho-da-Silva, A. Pio-Viana, and G. Ferreguetti. 2011. Seasonal and genetic influences on sex expression in a backcrossed segregating papaya population. Crop Breed. Appl. Biotechnol. 11:97-105.

Corrêa, P., T. Santana, F. Filho-Da-Silva, A. Pio, e M. Gonzaga. 2008. Comportamiento floral de híbridos de mamoeiro (Carica papaya L.) avaliados no verao e na primavera. Rev. Ceres 55:310-316.

Cruz, D.R., y A. Alcántara. 2000. Anormalidad floral en Phytolacca icosandra L. (Phytolaccaceae) en el Pedregal de San Ángel, México, D.F. Acta Bot. Mex. 53:27-33. 
Gil, A.I., y D. Miranda. 2005. Morfología de la flor y de la semilla de papaya (Carica papaya L.): variedad maradol e híbrido Tainug-1. Agron. Colomb. 23:217-222.

Liu, Z., P.H. Moore, H. Ma, C.M. Ackerman, M. Ragiba, Q. Yu, H.M. Pearl, M.S. Kim, J.W. Charlton, J.I. Stiles, F.T. Zee, A.H. Paterson, and R. Ming. 2004. A primitive Y chromosome in papaya marks incipient sex chromosome evolution. Nature 427:348-352.

López, G., y J. López. 1995. Introducción al micro SAS: Aplicación al análisis de experimentos agrícolas. CATIE, Turrialba, CRC.

Meyer, V.G. 1966. Flower abnormalities. Bot. Rev. 32:165-218.

Mirafuentes-Hernández, F., y F. Santamaría-Basulto. 2014. MSXJ, híbrido de papaya sin carpelodia para el sureste de México. Rev. Mex. Cienc. Agríc. 7:1297-1301.

Mora, E., y A. Bogantes. 1999-2002. Evaluación del potencial comercial de líneas S2 de papaya (Carica papaya) en la Región Atlántica de Costa Rica. Rev. Agric. Trop. 32:73-80.

Nakasone, H.Y. 1967. Papaya breeding in Hawaii. Agron. Trop. 17:391-399.

Nakasone, H.Y., and C. Lamoureux. 1982. Transitional forms of hermaphroditic papaya flowers leading to complete maleness. J. Am. Soc. Hort. Sci. 107:589-592.

Parés, J., C. Basco, y D. Jáuregui. 2002. Momento de antesis, dehiscencia de anteras y receptividad estigmática en flores de lechosa (Carica papaya) cv. Cartagena amarilla. Bioagro 14:17-24.

Parés, J., R. Linarez, M. Arizaleta, y L. Meléndez. 2004. Aspectos de la biología floral en lechosa (Carica papaya L.) cv. Cartagena roja en el estado Lara, Venezuela. Rev. Fac. Agron. Caracas 21:116-125.

Peres-Martelleto, L.A., R.L. Duarte-Ribeiro, M. Sudo-Martelleto, M.A. Da-Silva-Vasconcellos, e M. Ballestero-Pereira. 2011. Expressao da esterilidade femenina e da carpelodia em mamoeiro sob diferentes ambientes de cultivo protegido. Rev. Bras. Frutic. 33:1185-1193.

Rodríguez-Pastor, M.C. 2002. Consideraciones sobre la utilización de diferentes densidades en el cultivo de papaya (Carica papaya, L.) "Baixinho de Santa Amalia" en islas Canaría. Rev. Bras. Frutic. 24:707-710.

Ronse, D.L., and E.C. Smets. 1999. The floral development and anatomy of Carica papaya (Caricaceae). Can. J. Bot. 77:582598.

Silva, F., M. Gonzaga-Pereira, H.C. Cancela-Ramos, P. Corrêa-Damasceno, T.N. Santana, and C.D. Pereira. 2007a. Genotypic correlations of morpho-agronomic traits in papaya and implications for genetic breeding. Crop Breed. Appl. Biotechnol. 7:345-352.

Silva, F., M. Gonzaga-Pereira, P. Correa, T. Santana, A. Pio, R. Figueiredo, H. Cancela, and G. Ferreguetti. 2007b. Evaluation of the sexual expression in a segregating BHM3-4 papaya population. Crop Breed. Appl. Biotechnol.7:16-23.

Silva, F., M. Gonzaga-Pereira, H.C. Cancela-Ramos, P. Corrêa-Damasceno, N. Santana-Pereira, A. Pio-Viana, R. FigueiredoDaher, and G. Antônio-Ferreguetti. 2008. Estimation of genetic parameters related to morphoagronomic and fruit quality traits of papaya. Crop Breed. Appl. Biotechnol. 8:65-73.

Sippel, A.D., N.J. Claassens, and L.C. Holtzhausen. 1989. Floral differentiation and development in Carica papaya cultivar 'Sunrise Solo'. Sci. Hort. 40:23-33.

Storey, W.B. 1953. The genetics of the papaya. J. Hered. 44:70-78.

Storey, W.B. 1967. Theory of the derivations of the unisexual flowers of Caricaceae. Agron. Trop. 17:273-321. 
Terra-De-Almeida, F., C. Sales, E. Fernández, and S. Gripa. 2003. Sexual expression of papaya tree affected by depth of irrigation in the North Region of Rio de Janeiro, Brazil. Rev. Bras. Frutic. 25:382-385. doi:10.1590/S0100-29452003000300005

Vázquez, E., E. Román, y R. Ariza. 2008. Fenología y unidades calor de genotipos de papayo en el sur de Tamaulipas, México. Rev. Fitotec. Mex. 31:45-48. 